\title{
3D Chemistry: How 3D printing could help embed crystallography within chemistry undergraduate teaching
}

$\underline{\text { Peter A. Wood }}^{1}$, Amy A. Sarjeant ${ }^{2}$, Ian J. Bruno ${ }^{1}$, Clare F. Macrae ${ }^{1}$, Helen E. Maynard-Casely ${ }^{3}$ and Matthew Towler ${ }^{1}$

${ }^{1}$ The Cambridge Crystallographic Data Centre, 12 Union Road, Cambridge, CB2 1EZ, UK.

${ }^{2}$ The Cambridge Crystallographic Data Centre, 174 Frelinghuysen Rd. Piscataway, NJ 08854, USA.

${ }^{3}$ Australian Nuclear Science and Technology Organisation, Locked Bag 2001, Kirrawee DC, Australia.

\section{E-mail: wood@ccdc.cam.ac.uk}

Chemistry is inherently three-dimensional in nature - only a very small fraction of compounds, mostly aromatic ones, are essentially flat and even those are not perfectly two-dimensional. Despite this, much of chemistry undergraduate teaching is still done using lecturing and textbooks, which by nature can only deliver 2D diagrams, albeit often using hashed or wedged bonds to try to help convey the 3D geometry. Studies have shown that students who are engaged in active learning, including hands-on activities or group-based discussions, have better learning rates than their peers whose sole source of knowledge is through textbooks and passive lectures. ${ }^{1}$ In this spirit, the CCDC has sought to help augment classroom education by introducing a tactile component through 3D printed molecules.

The Cambridge Structural Database (CSD) ${ }^{2}$ contains over 875,000 structures of molecules ranging from simple organic molecules to complex metal-organic frameworks, and everything in between. Structures can be found in the CSD to illustrate many concepts within chemistry curricula, such as molecular structure \& bonding (incl. VSEPR theory), molecular point group symmetry, intermolecular interactions and reaction pathways.

Allowing students to handle these structures, to explore them not only visually but also tactilely, we can stimulate a deeper understanding of learning outcomes such as the connection between structure and function or spectroscopic signatures. The use of 3D printed molecular models obtained directly from crystallography within chemistry education also helps to emphasise the value of crystallography and crystallographic data throughout chemistry as well as putting real experimental results in students' hands. This presentation will focus on several ways in which 3D models from crystal structures can enhance chemistry education for students of all levels.

(1) Freeman, S.; Eddy, S. L.; McDonough, M.; Smith, M. K.; Okoroafor, N.; Jordt, H.; Wenderoth, M. P. Active learning increases student performance in science, engineering, and mathematics. Proc. Natl. Acad. Sci. U. S. A. 2014, 111, 8410-8415. DOI: 10.1073/pnas.1319030111.

(2) Groom, C. R.; Bruno, I. J.; Lightfoot, M. P.; Ward, S. C., The Cambridge Structural Database. Acta Cryst. B, 2016, 72, 171-179. DOI: 10.1107/S2052520616003954. 